\title{
SOBRE ALGUNAS GELIDIACEAS NUEVAS O POCO CONOCIDAS DE LAS COSTAS ESPAÑOLAS
}

\author{
J. SEOANE - CAMBA *
}

\section{RESUMEN:}

En esta comunicación damos a conocer una nueva localidad de la isla de Tenerife para Gelidiella tinerfensis J. Seoane-Camba, e igualmente se cita Gelidiella tenuissima J. Feldmann y G. Hamel en San Vicente de La Barquera (Santander) como nueva localidad para las costas españolas.

Se hace un estudio comparativo de Gelidium cartilagineum (L.) Gaillon, procedente de El Cabo, y Gelidium cartilagineum (L.) Gaillon var. canariensis Grunow de Tenerife, llegando a la conclusión de que las posibles afinidades morfológicas e histológicas entre ambos taxa no existen, o son tan lacias como las que pueden existir entre cualesquiera especies de Gelidium. Por ello proponemos el nombre de Gelidium canariensis (Grunow) nov. sp. para la planta canaria.

Finalmente se describe una nueva especie de Gelidium, encontrada en la costa cantábrica de la Península Ibérica, con el nombre de Gelidium cantabricum nov. sp.

\section{SUMMARY:}

In this paper a new locality of Tenerife Island is made known for Gelidiella tinerfensis J. Seoane-Camba. Likewise, San Vicente de La Barquera (Santander) is made known as a new locality in the spanish shore for Gelidiella tenuissima J. Feldmann y G. Hamel.

On the other hand a comparative study between Gelidium cartilagineum (L.) Gaillon, from El Cabo, and Gelidium cartilagineum (L.) Gaillon var. canariensis Grunow, from Teneri$\mathrm{fe}$, is made. We conclude that both taxa are different species and we propose the name Gelidium canariensis (Grunow) nov. sp. for the canary plant.

Finally we describe a new species of Gelidium, that has been find at Cantabria, with the name Gelidium cantabricum nov. sp.

Gelidiella tinerfensis ha sido descrita por nosotros a partir de unos ejemplares recogidos en diciembre de 1972 en los alrededores del Puerto de La Cruz (Tenerife), en la parte superior de una pequeña gruta batida, abierta y bien expuesta al oleaje. 
Es una planta de 1 a 1,5 cm., y se presenta formando un césped de color rojo oscuro. Está formada por filamentos horizontales radicantes entrelazados los unos con los otros y fijados por rizoides. Los filamentos radicantes son siempre cilíndricos, de 70-85u. de diámetro, sin ricinas en su interior y con células superficiales dispuestas irregularmente. Estos filamentos radicantes producen filamentos erguidos, cilíndricos en la parte inferior y más o menos comprimidos hacia la parte media, donde alcanzan generalmente el mayor diámetro, de hasta 200 micras. La ramificación es más bien irregular y en los extremos de los ejes y ramas frecuentemente se arquean y llevan, a veces, rizoides para la fijación por su parte apical. Los ejes erguidos carecen tabién de ricinas y crecen por una célula apical. Las células superficiales se disponen igualmente en forma irregular. Las tetrasporas se disponen en forma bastante regular en uve y pueden presentarse en estiquidos más o menos normales, pero también es frecuente que sean ramificadas con una parte vegetativa terminal que puede proseguir su crecimiento. No se han observado órganos sexuales ni cistocarpos. (Seoane-Camba, 1977).

Cuando esta planta fue recogida en 1972, un poco al azar debido a su pequeño tamaño, y examinada en el laboratorio de Barcelona, me pareció interesante y pasó al paquete de problemas a resolver, cuando se dispusiera de más material. Pero la espera se prolongó hasta agosto de 1975, en que, en colaboración con el Instituto de Química Orgánica de La Laguna, recorrimos las islas de Tenerife y Fuerteventura buscando especies de Laurencia. Cuando correspondía la visita al Puerto de La Cruz y me dispuse a recoger la planta en el punto donde había sido encontrada anteriormente, tal localidad había desaparecido, puesto que por causas quizá naturales pero muy probablemente artificiales, se había cubierto de piedras y arena. La playa próxima se había extendido más lejos y la pequeña cueva, que tanto significaba para mí, había desaparecido. En vista de esto decidí describir la especie a partir del poco material de que disponía, puesto que estaba convencido de que se trataba de una especie nueva; se trata de un ejemplo típico de lo que puede ocurrir continuando con esta progresión geométrica en la velocidad de cambio, transformación y destrucción que se está operando en la naturaleza; con ello me exponía a describir una especie cuando podía darse el caso de no encontrarla más, es decir, describirla "post morten". El trabajo lo presenté aprovechando la reunión extraordinaria de la Sociedad Ficológica de Francia dedicada en homenaje al Dr. Feldmann que se realizó en 1976. Por eso pongoespecial énfasis en dar aquí una nueva localidad para esta especie.

En la enorme cantidad de material recogido en 1975, mucho del cual está todavía sin estudiar, hace un par de meses le ha correspondido el turnoal obtenido en la localidad de Majanicho (algunos kilómetros al este del Puerto de La Cruz) y allí he encontrado, afortunadamente, Gelidiella tinerfensis J. Seoane-Camba, que será por lo tanto la segunda localidad para la isla de Tenerife y para mí un alivio.

Otra cita que considero interesante dar a conocer aquí es la relacionada con la especie de Gelidiella tenuissima J. Feldmann y G. Hamel, en las costas de Santander.

Se trata de una especie de Gelidiella mucho más pequeña, que he encontrado en los acantilados de San Vicente de La Barquera en agosto de 1971 y que he vuelto a encontrar en septiembre de 1978. (Fig. 1). 


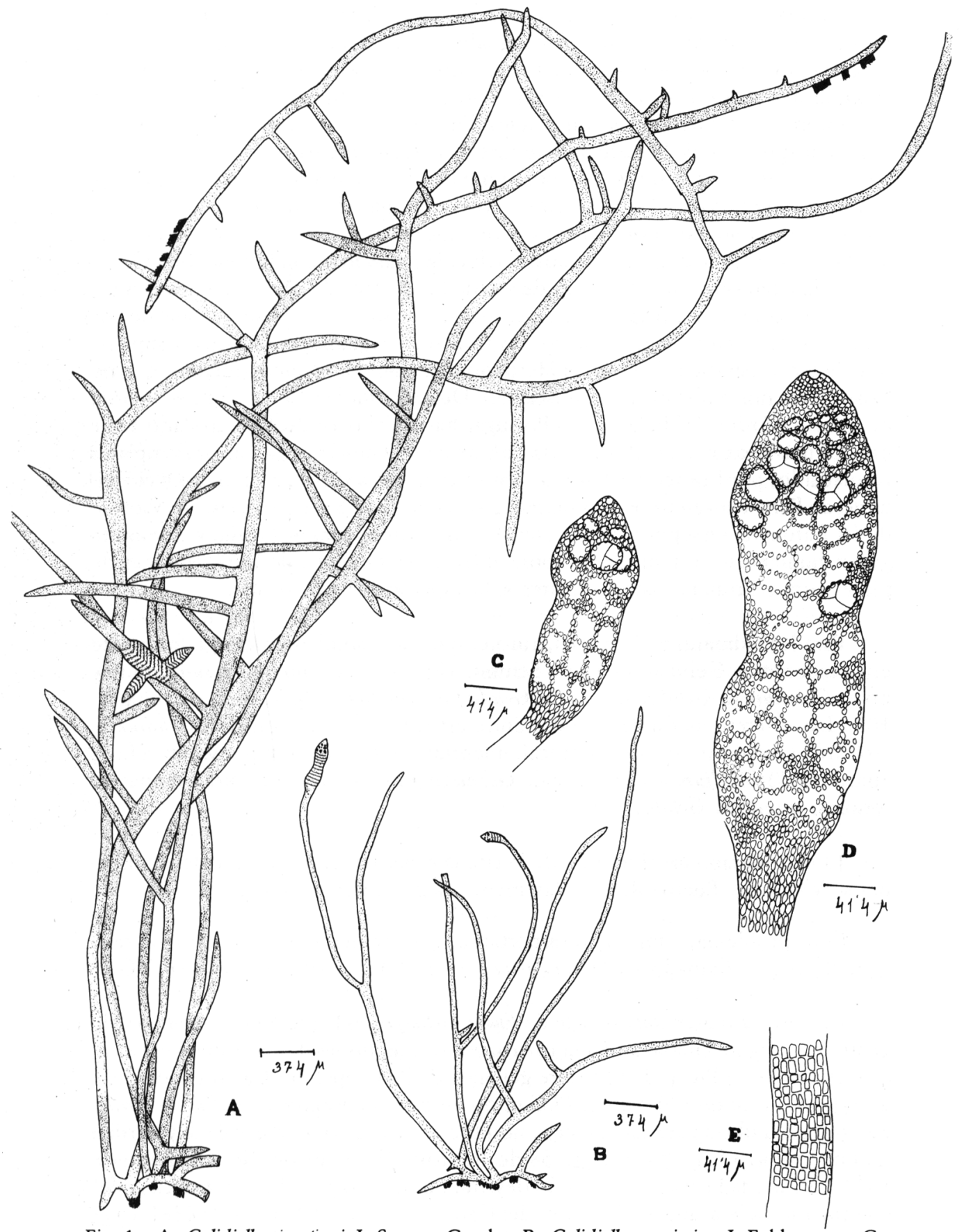

Fig. 1.-A, Gelidiella tinerfensis J. Seoane-Camba; B, Gelidiella tenuissima J. Feldmann y G. Hamel; C y D, estiquidios de Gelidiella tenuissima J. Feldaman y G. Hamel; E, células superficiales de Gelidiella tenuissima J. Feldmann y G. Hamel. 
Es una planta que, como la especie anterior, forma un césped de color rojo más o menos oscuro, con filamentos igualmente radicantes e intrincados, fijos al sustrato por rizoides abundantes y forma ramas relativamente poco ramificadas y finas, de alrededor de 42 a 75 micras de diámetro y de altura hasta $5 \mathrm{~mm}$. La estructura interna carece de ricinas tanto en los filamentos basales como en los eréctiles, pero la disposición de las células exteriores, casi cuadrangulares o rectangulares, es bastante regular, formandoseries longitudinales y de un diámetro aproximado de 4 micras por término medio. El crecimiento es por una célula terminal bien ostensible. Los etiquidios son normalmente terminales y las tetrásporas manifiestan una tendencia a colocarse en líneas transversales algo curvadas con la concavidad hacia arriba. Las tetrásporas tetraédricas de alrededor de 16 a 20 micras como máximo.

Esta planta concuerda perfectamente con los esquemas y descripciones que de Gelidiella tenuissima hacen J. Feldmann y G. Hamel e igualmente con los ejemplares que de dicha especie existen en el Herbario de París; por lo que estimamos que no existe la menor duda en su identificación. Donze (1968) cita esta especie en la ría de Arosa; y Ardré (1970) la cita en Portugal, hasta Cabo Verde. Por otro lado, la localidad más próxima hacia el E. es la de Biarritz en Francia, según unos ejemplares recolectados por Bornet que figuran como Gelidium tenuissimum Thuret. En el Herbario de París existen unos ejemplares recolectados por Sauvageau en San Vicente de La Barquera, en septiembre de 1893 de Gelidium pannosum Grunow, que no figuran siquiera en su publicación sobre el Golfo de Gascuña de 1897, cuyos ejemplares son, a primera vista, similares a la especie que nos ocupa.

Para J. Feldmann y G. Hamel, autores que han estudiado monográficamente estas especies de Gelidiáceas y para Schmitz (1895), el Gelidium pannosum Grunow no es Gelidium ni Gelidiella, sino un Gelidiopsis (ver Rev. Algl. T. IX, fasc. 1-2, pág. 102). Las diferencias fundamentales entre estos tres géneros son: $1 .^{\circ}$ ) Gelidium y Gelidiella crecen por una célula apical, mientras Gelidiopsis crece por varias células apicales..$^{\circ}$ ) Gelidium se diferencia de Gelidiella porque el primero posee ricinas interiores, mientras Gelidiella no.

La distribución en el mundo de la citada especie incluye localidades de Indonesia, VietNam, Puerto Rico, y las mencionadas anteriormente.

Otro problema, a mi juicio importante, que me permito presentar es el relacionado con el llamado Gelidium cartilagineum (L.) Gaillon var. canariensis Grunow.

Gelidum cartilagineum procede de Fucus cartilagineus L. (de Species plantarum de 1630), y así figura citada la planta canaria en "Essais sur les Iles Fortunées et l'antigue Atlantide ou Précis de l'histoire générale de l'Archipel des Canaries» de Bory de Saint Vincent de 1803. En 1828 Gaillon propone el nombre de Gelidium cartilagineum (L.) Gaillon para esta especie, nombre utilizado por Barker-Webb y Berthelot en el tomo 3. ${ }^{\circ}$, 2. $^{\text {a }}$ parte, pág. 158 de «Histoire Naturelle des Iles Canaries» de 1840. La variedad canariensis ha sido descrita por Grunow en Picone "Crociera del corsaro alle isole Madera e Canarie» del Capitano Enrico d'Albertis de 1881, cuya descripción es la siguiente: "Differt a planta capensi caule humiliore, sursum subfastigiatim ramoso, pinnis brivioribus. Habitu formas nonnullas Gelidii glandulifolii aemulat et formas illas capenses, quas Kützing sub nomine Gelidii rigidi et Gelidii asperi desinxit 
quae autem ab illis speciebus diversissimae, et Gelidii cornei varietates sistem». El material original sobre el cual ha hecho Grunow su descripción no he podido consultarlo. Sin embargo en Borgesen "Marine algae from the Canary Islands", de 1925, se hace referencia a tal descripción y se presenta una figura bien típica de la planta, que corresponde exactamente con el material que se encuentra en el Herbario de París, como Gelidium cartilgineum Gaillonvar. canariensis Grunow y con el material que en gran cantidad hemos tenidola oportunidad de recoger en la isla de Tenerife, cerca del Puerto de La Cruz.

Ya en la breve descripción de la planta que hace Borgesen, se dice que «comparadas con las plantas procedentes de El Cabo, los ejemplares canarios tienen una apariencia más corimbiforme y subfastigiada, la ramificación es más irregular con ramas de muy variada longitud, se podría pensar por ello que se ha perdido la fina ramificación pinnada de la forma típica, mientras que el eje principal bien desarrollado y ostensible en la forma típica no es tan marcado en la planta canaria». Pero, en mi opinión, las diferencias entre el Gelidium cartilagineum. (L.) Gaillon, típico y el llamado Gelidium cartilagineum (L.) Gaillon var. canariensis, van mucho más lejos, hasta concluir que las posibles afinidades morfológicas e histológicas entre ambos taxa no existen, o son tan lacias como las que pueden existir entre cualesquiera otras especies de Gelidium.

Con esta idea me he propuesto hacer un estudio comparativo entre elllamado G. cartilagineum (L.) Gaillon var. canariensis Grunow, tomando como material de estudio el recogido en Tenerife en diciembre de 1972 y agosto de 1975 por nosotros mismos y el G. cartilagineum (L.) Gaillon de El Cabo de Buena Esperanza, tomando como material de estudio el enviado desinteresadamente por «Novogel» de Santander, a cuya firma agradezco desde estas líneas su colaboración.

El análisis comparativo se ha extendido fundamentalmente al color, a las dimensiones y morfología general de la planta, a la disposición y dimensiones de las células superficiales e internas, a la morfología de los estiquidios y a la disposición y dimensiones de las tetrasporas.

El color de estas plantas es bien diferente; Gelidium cartilagineum (L.) Gaillon, posee un color rojo, si bien algo oscuro, resalta fácilmente como alga roja, que és; mientras el llamado Gelidium catilagineum (L.) Gaillon, var. canariensis Grunow presenta un color oscuro, prácticamente negro, como el mismo Sauvageau hace resaltar en su trabajo "A propos de Cystoseira" pág. 99, en donde da la siguiente descripción: "La Cystoseira abies marina habita las rocas batidas de la zona inferior. Cuando crece sobre una pared vertical, opuesta al mar, se observa siempre la sucesión siguiente, tan ostensible que se distingue a distancia: una banda amarilla, formada por ella, de 20-40 cm. de altura; inmediatamente, por debajo, una banda de color rojo violáceo de Gelidium arbuscula Bory; por debajo de ésta y prolongándose en la parte noaccesible y no visible una banda más ancha oscura, casi negra de Gelidium cartilagineum Gaillon». De lo que podemos inferir que la misma composición química de los pigmentos es diferente.

Si comparamos la morfología y el porte de la planta vemos que Gelidium cartilagineum (L.) Gaillon, posee una tendencia muy marcada hacia la ramificación pinnada, especialmente ostensible en las últimas pínnulas que se hallan relativamente 
bien ordenadas; por otra parte existe una diferencia muy marcada en cuanto al diámetro y robustez entre el eje principal de los diferentes órdenes. En cambio, en la llamada variedad canariensis, Grunow la ramificación es muy diferente, mucho menos regular y con una mayor tendencia a una estructura corimbiforme sin diferencias especialmente marcadas entre eje principal, ramas, pinnas y pínnulas. (Fig. 2).
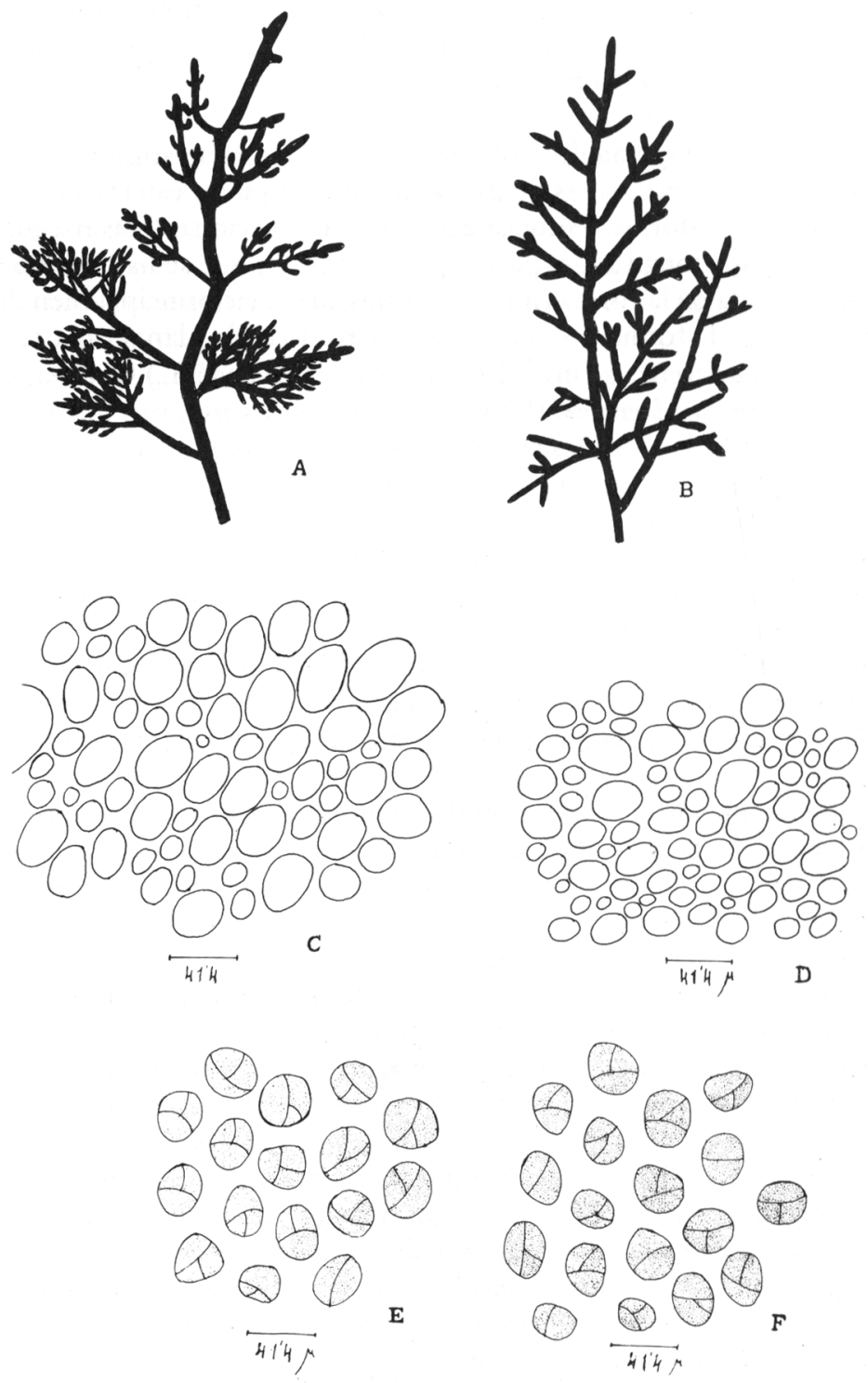

Fig. 2.- A y B, silueta esquemática de la ramificación de Gelidium cartilagineum(L.) Gaillon (A) y Gelidium cartilagineum (L.) Gaillon var. canriensis Grunow (B); C y D, células internas (sección transversal) de especie y variedad respectivamente; E y F, tetrasporas de las mismas respectivamente. 
En cuanto a las dimensiones de las plantas son también muy diferentes, algunos ejemplares de G. cartilagineum (L.) Gaillon de que disponemos (relativamente pocos) pueden sobrepasar los $45 \mathrm{~cm}$. y el diámetro del eje principal puede ser mayor a $3 \mathrm{~mm}$.; mientras los ejemplares de la llamada var. canariensis Grunow de que disponemos (que son varios cientos) ninguno sobrepasa los $30 \mathrm{~cm}$., teniendo como término medio $25 \mathrm{~cm}$., y el diámetro deleje principal es por término medio de 1 a $1,5 \mathrm{~mm}$. (Fotos 1 y 2 ).

Otra diferencia a mi juicio importante es la disposición de las tetrasporas en los estiquidios o receptáculos esporíferos. En ambas. plantas los estiquidios son alargados y espatulados. En G. cartilagineum (L.) Gaillon presentan normalmente un margen estéril que rodea la parte esporífera casi completamente, excepto en el ápice; mientras en la llamada var. canariensis Grunow ese margen no existe o en otros casos recubre sólo la base de la parte fértil, especialmente en los jóvenes. (Fig. 3).

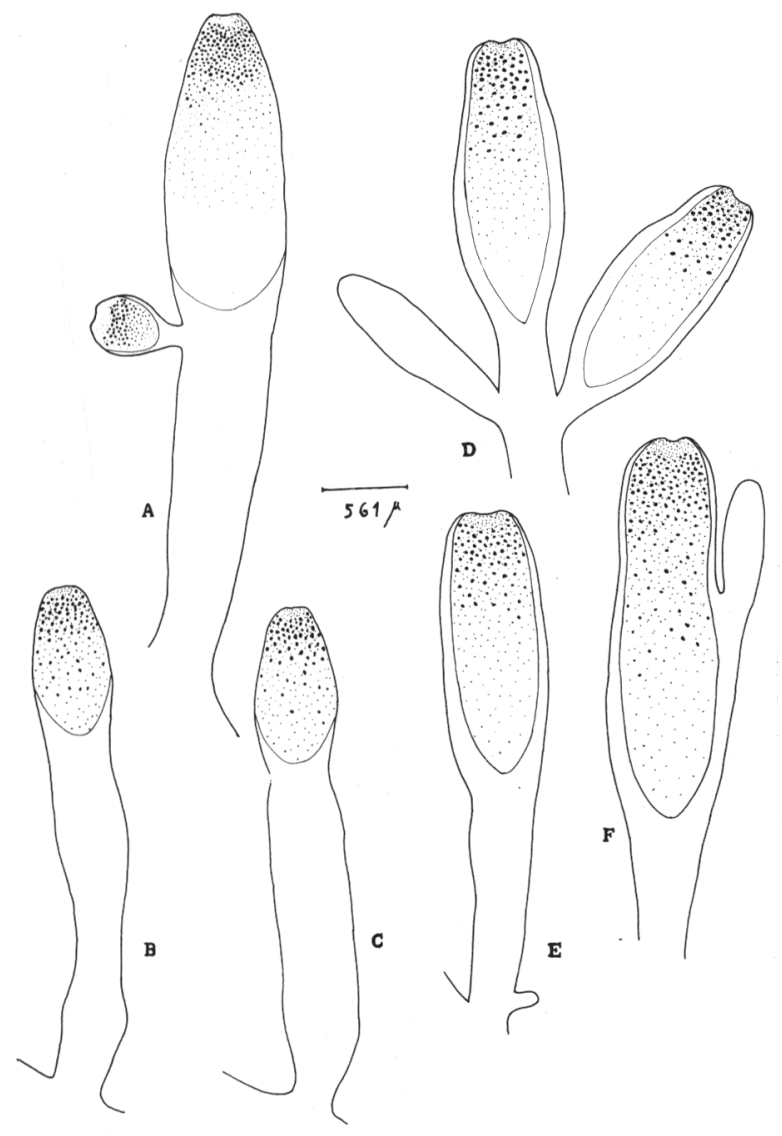

Fig. 3.- A, B y C, estiquidios tetrasporíferos de Gelidium cartilagineum (L.) Gaillon var. canariensis Grunow; D, E y F, estiquidios tetrasporíferos de Gelidium cartilagineum (L.) Gaillon. 
El tamaño de las tetrasporas no resulta muy demostrativo puesto que sus dimensiones dependen de su estado de madurez. (Fig. 2).

La estructura interna general del talo es típicamente de Gelidium en ambos casos, con una zona cortical de células dispuestas en series moniliformes perpendiculares a la superficie, y debajo una zona periférica de ricinas que continúa con una parte central de células más grandes. Si comparamos las dimensiones de las células internas entre ambas plantas vemos que las de G.cartilagineum(L.) Gaillon son mayores que las del llamado var. canariensis, Grunow del orden de 63 contra 40 micras respectivamente, como máximo observado. (Fig. 2). Igualmente las dimensiones de las células superficiales, tanto en la parte apical como en la basal de ambas plantas son diferentes, siendo mayores en $G$. cartilagineum (L.) Gaillon y menores en la var. canariensis, Grunow del orden de 5 contra 4 micras respectivamente, como término medio. Por otra parte, la disposición de las células corticales, es mucho más ordenada en $G$. cartilagineum (L.) Gaillon típico, especialmente en las partes jóvenes. (Fig. 4).

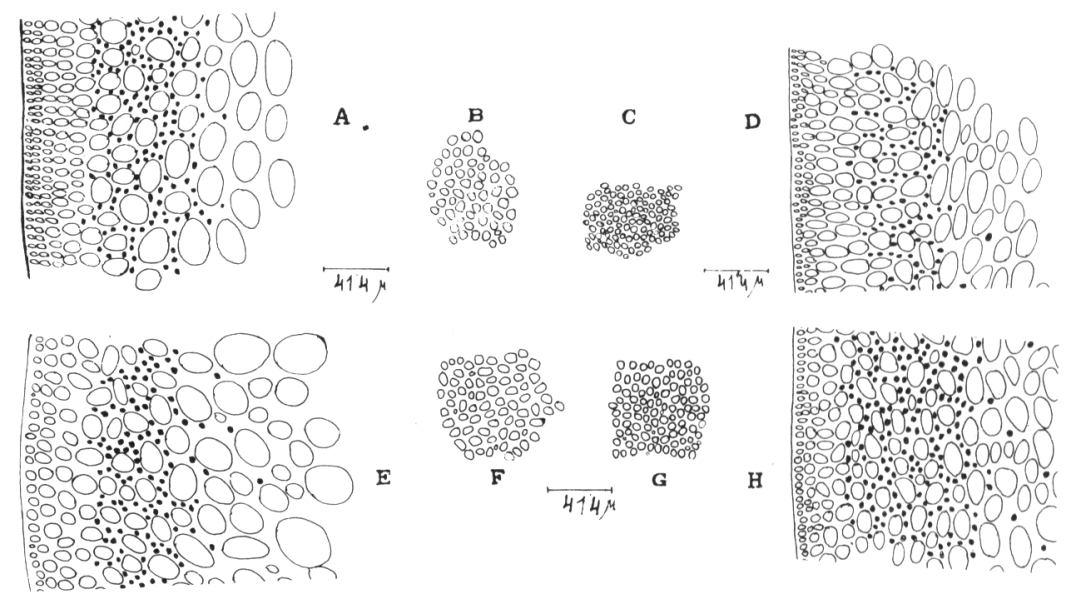

Fig. 4.- Comparación esquemática de las células superficiales y sección transversal de la parte apical (B y A) y parte basal (F. y E) de Gelidium cartilagineum (L.) Gaillon, así como de la parte apical (C y D) y parte basal ( $\mathrm{G}$ y H) de Gelidium cartilagineum (L.) Gaillon var. canariensis Grunow.

Por todo lo que acabamos de decir, llegamos a la conclusión de que las posibles afinidades morfológicas e histològicas y probablemente bioquímicas (a nivel de pigmentos al menos), entre las plantas estudiadas no existen o si existen son tan lacias como puedan ser las existentes entre cualesquiera especies de Gelidium, como ya he dicho anteriormente. Por ello estamos convencidos de que el llamado G. cartilagineum (L.) Gaillon var. canariensis Grunow no puede considerarse como perteneciente a la especie Gelidium cartilagineum, (L.) Gaillon sino que se trata de una especie diferente y como tal debe ser considerada. En consecuencia, proponemos el nombre de Gelidium canariensis (Grunow) sp. nov. para la planta canaria. 
Por último, también creo de interés describir aquí una nueva especie encontrada en la costa cantábrica de la Península Ibérica, perteneciente igualmente al género Gelidium.

Se trata de una planta que existe en muy poca cantidad, en profundidades próximas a los 10 metros en la zona de Santander, y mezclada con Gelidium sesquipedale (Clem.) Thuret. Su tamaño puede alcanzar los $40 \mathrm{~cm}$. El color es rojo oscuro en fresco, un poco más oscuro, por lo tanto, que el de G. sesquipedale (Clem.) Thuret y casi negro en seco. El talo es cilíndrico o muy poco comprimido, tanto en el eje principal como en sus ramificaciones, cuyo diámetro, de alrededor de $1,5 \mathrm{~mm}$., no se diferencia fundamentalmente del eje principal. La ramificación es muy irregular, con ramas raramente opuestas a veces unas cuantas secundas, y con frecuencia ocurre, especialmente hacia las partes distales del eje o de las ramas, que 4, 5 ó 6 de ellas salen prácticamente del mismo punto. (Foto 3). La estructura interna es típica del género Gelidium, con un córtex de células moniliformes y bastante bien ordenadas, dispuestas de forma perpendicular a la superficie; debajo una capa con abundancia de ricinas, y en el interior células más grandes. Si comparamos su estructura con la de G. sesquipedale, (Clem.) Thuret, vemos que la zona cortical de células moniliformes es un poco más gruesa y su ordenación es también más acentuada. Las células superficiales, de alrededor de 3,7 a 4,1 micras de diámetro, son también más pequeñas que las de G. sesquipedale (Clem.) Thuret. (Fig. 5).
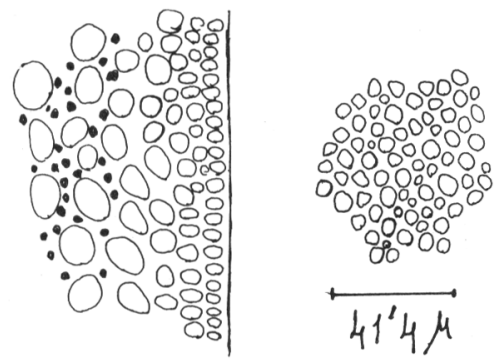

A
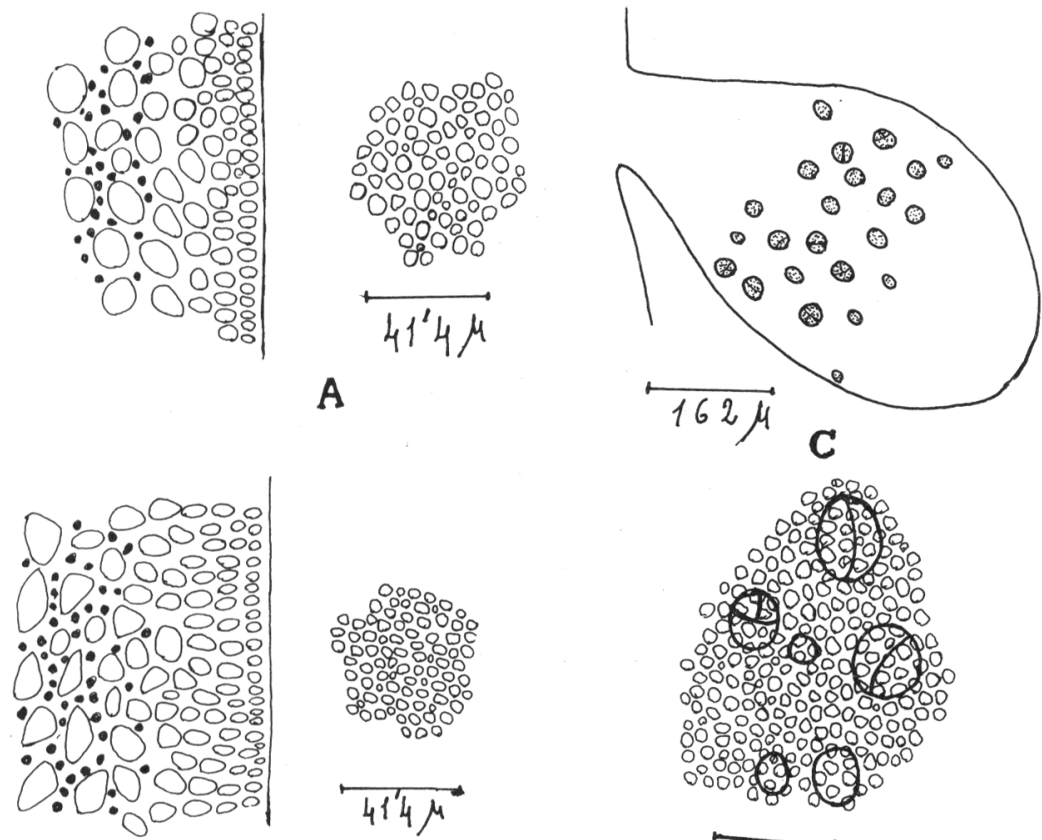

B

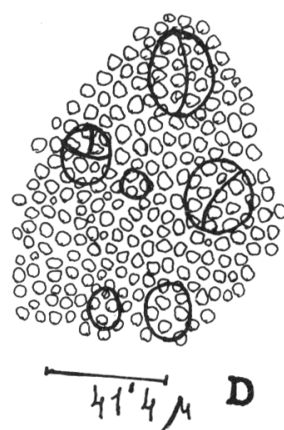

Fig. 5.- A, sección transversal y células superficiales de Gelidium sesquipedale (Clemen.) Thuret; B, sección transversal y células superficiales de Gelidum cantabricum nov. sp.; C, estiquidio tetrasporifero de Gelidium cantabricum nov. sp.; D, células superficiales y tetrasporas de Gelidium cantabricum nov. sp. 
No se han encontrado plantas con órganos sexuales ni cistocarpos, en cambio sí tetrasporíferas. Las tetrasporas se forman en estiquidios pequeños, casi sentados, muy poco numerosos, que a primera vista semejan diminutas ramitas o incluso pequeñas verrugas, y que se forman hacia la mitad de ciertas ramas. Las tetrasporas, tetraédricas, tienen un diámetro variable según su madurez, pero las mayores observadas pueden llegar a 28-30 micras.

Por todos estos caracteres, estamos convencidos que nos encontramos ante una especie que la literatura no ha descrito todavía. Por ello proponemos para dicha especie el nombre de Gelidium cantabricum nov. sp. por haber sido encontrada por primera vez en Cantabria.

\section{DIAGNOSE}

Gelidium cantabricum nov. sp.

Frons teres, rubro-obscura, exsiccatione rubro-nigrescenti, $40 \mathrm{~cm}$. alta et $15 \mathrm{~mm}$. lata. Rami irregulariter nonnunquam subfasciculatum dispositi. Structura generis. Cellulae corticales ad 3,7-4,1 u. latae, irregulariter dispositae.

Tetrasporangia tetraedrica, ad 28-30u. lata in stichidis clavalis, minutissimis, ad ramulorum lateralium. Anteridia et cystocarpia ignota. Legi in infralittoralibus apud Cantabria.

\section{AGRADECIMIENTOS}

Debo manifestar nuestro agradecimiento al Instituto de Química Orgánica de la Universidad de La Laguna (Tenerife) por su colaboración en la recolección de las algas de Canarias; a la firma Novogel, S.A. por su amable envío de material de Africa del Sur y al delegado en Llanes de la firma Hispanagar, S.A., por su amable colaboración en la obtención de ejemplares de Gelidium cantabricum nov. sp.

\section{BIBLIOGRAFIA}

ARDRE, F. 1970. Contribución a l'étude des algues marines du Portugal. Portugalie Acta Biol. (B) 10, nos. 1/4.

BAARDRETH, E. 1941. The marine algae of Tristán da Cunha. Norreegian Scient. exped. to Tristan da Cunba, n. 9.

BARKER-WEBB y BERTHELOT, S. 1840. Histoire Naturell des Iles Canaries. Béthune, Paris.

BLANQUIST, H. L. y ALMODOVAR, L.R. 1961. The occurence of Gelidiella tenuissima Feldmann et Hamel in Puerto Rico. Nov. Hedw., 3, 1.

BORNET, E. y THURET, G. 1876. Notes algologiques. Bibliotheca Pbycologica, 9. Reprint 1969. 
BÖRGESEN, F. 1927. Marine Algae from the Canary Islands. III, part. 1. Kgl. Danske Videnskab Selskab. Biol. Meddel. VI, 6.

BORY DE SAINT VINCENT 1803. Essais sur les îles Fortunées et l'antigue Atlantide, ou Précis de l'histoire générale de d'Archipel des Canaries. Baudouin. Paris.

CHAPMAN, V. J. 1963. The Marine Algae of Jamaica. The Inst. of Jamaica. Scient. Series $n .{ }^{\circ} 12$.

DE TONI, G. B. 1889-1924. Sylloge algarum. Vol. 1-6. Padua.

DIXON, P.S. 1958 a. The occurence of Gelidium sesquipedale (Clem.) Thuret. in the British Isles. Brit. Phycol. Bull. 1, 6.

1958 b. The structure and developement of the tallus in the Brithish species of Gelidium and Pterocladia. Ann. of Bot. N. S. 22, 87.

1961. On the classification of Florideae with particular reference to the position of the Gelidiaceae. Botánica Marina. Vol. III, fasc. 1.

DIXON, P. S. y IRVINE, L. M. 1978. Seawees of the British Isles. British Museum. Londres.

DONZE, M. 1968. The algae vegetation of the Ria de Arosa (N. W. Spain). Blumea, 16.

FELDMANN, J. 1931. Remarques sur les genres Gelidium Lamour., Gelidiopsis Schmitz et Echinocaulon (Kütz.) emend. Travaux cryptogamiques dédis a Louis Mangin. Paris.

FELDMANN,J. y HAMEL, G. 1934. Observations sur quelques Gelidiacées. Rev. Gen. Bot. 46. 1936. Floridées de France. VIII, Gelidiales, Rev. Algol. 9, 1-2.

JOHNSTON, C.S. 1969. Studies on the ecology and primary production of Canary Islands marine algae. Proc. Int. Seawees Symp. 6. Santiago de Compostela.

KYLIN, H. 1956. Die Gattungen der Rhodophyceen. Lund.

KÜTZING, F. T. 1843. Phycologia Generalis. Leipzig. 1845-1869. Tabulae Phycologicae. Nordhausen.

MARTIN AGUADO, M. 1957. Las algas de Canarias en la obra de Viera y Clavijo. Rev. de Historia canaria. T. XXIII.

OKAMURA, K. 1901-34 Icones of Japanese Algae. Tokyo. T. 1-7. 1934. On Gelidium and Pterocladia of Japan. Jour. of Imp. Fischer. Instit. Vol. 29, n. ${ }^{\circ} 2$.

PICONE, A. 1881. Alghe "Crociera del corsario alle isole Madera e Canarie» del Capitano Eurico d'Albertis. Genova.

SAUVAGEAU, C. 1897. Note preliminaire sur les algues marines du Golfe de Gascogne. Journ. de Botanique, 11.

SCHMITZ, Fr. 1895. Marine Florideen von Deutsch-Ostafrika. Engler's Bot. Jabrb. 21.

SEOANE-CAMBA, J. 1971. Sur une nouvelle espéce de Gelidiella trouvée aux Iles Canaries: Gelidiella tinerfensis nov. sp. Bull. Soc. Phycol. de France, número 22.

TAYLOR, W. R. 1928. The marine Algae of Florida. Bibliotheca Phycologica. B. 2. Reprint 1967. 1972. Marine algae of the Eastern Tropical and Subtropical coasts of Americas. The University of Michigan Press. 




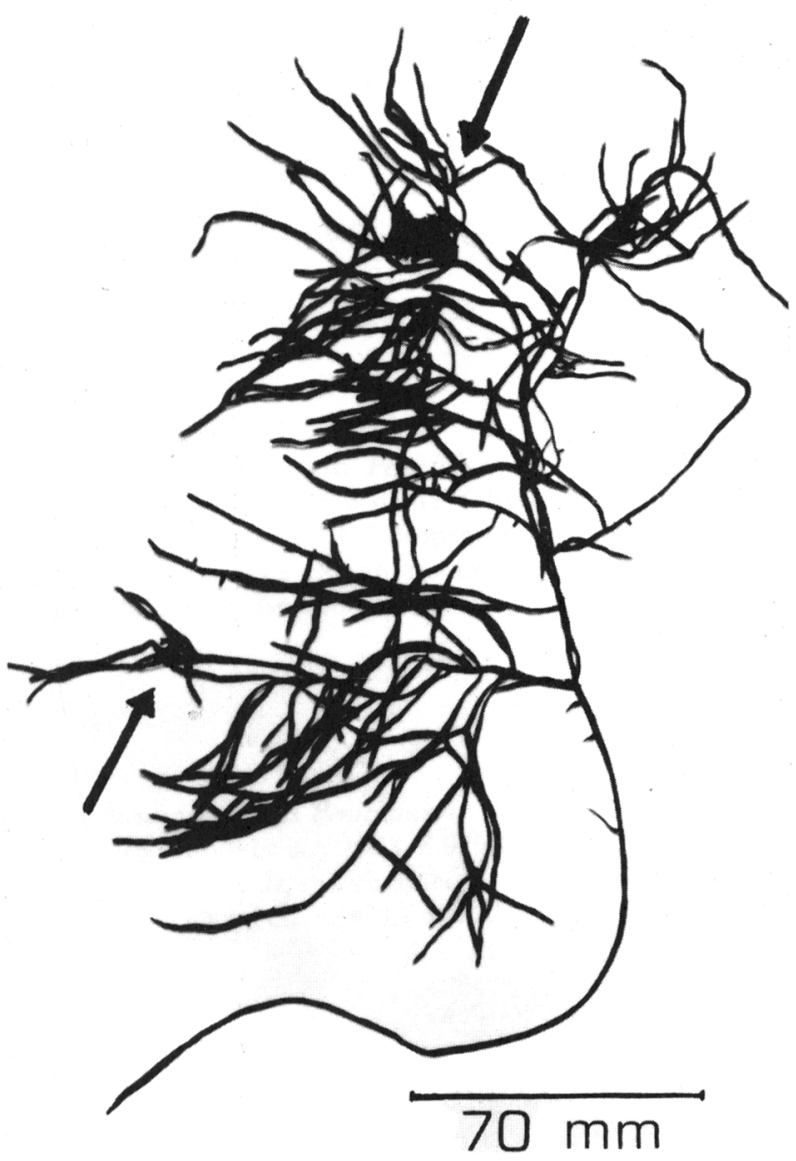

FOTO 3

Gelidium cantabricum nov. sp. 